\title{
How Spatial Layout, Interactivity, and Persistent Visibility Affect Learning with Large Displays
}

\author{
Eric D. Ragan, Alex Endert, Doug A. Bowman, and Francis Quek \\ Virginia Tech \\ Department of Computer Science \\ Center for Human-Computer Interaction \\ 2202 Kraft Drive, Blacksburg, VA 24060, USA \\ \{eragan | aendert | dbowman | quek\} @vt.edu
}

\begin{abstract}
Visualizations often use spatial representations to aid understanding, but it is unclear what properties of a spatial information presentation are most important to effectively support cognitive processing. This research explores how spatial layout and view control impact learning and investigates the role of persistent visibility when working with large displays. We performed a controlled experiment with a learning activity involving memory and comprehension of a visually represented story. We compared performance between a slideshow-type presentation on a single monitor and a spatially distributed presentation among multiple monitors. We also varied the method of view control (automatic vs. interactive). Additionally, to separate effects due to location or persistent visibility with a spatially distributed layout, we controlled whether all story images could always be seen or if only one image could be viewed at a time. With the distributed layouts, participants maintained better memory of the associated locations where information was presented. However, learning scores were significantly better for the slideshow presentation than for the distributed layout when only one image could be viewed at a time.
\end{abstract}

\section{Categories and Subject Descriptors}

H.5.2 [Information Interfaces and Presentation]: User Interfacesgraphical user interfaces (GUI).

\section{General Terms}

Experimentation, Human Factors

\section{Keywords}

Large displays, learning, memory, interactivity, use of space

\section{INTRODUCTION}

Learning is a complicated phenomenon involving sense-making through the organization of many simple units of information [1]. Due to their many successes in making information easier to understand, visualizations are commonly used to help viewers learn new information [6]. Visualization and presentation tools often

Permission to make digital or hard copies of all or part of this work for personal or classroom use is granted without fee provided that copies are not made or distributed for profit or commercial advantage and that copies bear this notice and the full citation on the first page. To copy otherwise, to republish, to post on servers or to redistribute to lists, requires prior specific permission and/or a fee.

AVI '12, May 21-25, 2012, Capri Island, Italy

Copyright (c) 2012 ACM 978-1-4503-1287-5/12/05... \$10.00. use spatial representations to present information or show relationships between different visual components [12]. For example, intelligence analysis tools help analysts to make sense of large information sets by looking through clustered documents [28]. In a school setting, a student can use a linear strip of thumbnail previews to help keep track of the PowerPoint slides while studying. For these types of spatial information presentations, in which information is mapped to different locations, previous work has provided evidence that learners can refer to locations to help recall the associated information $[9,22,23]$.

The types of possible spatial information presentations are heavily dependent on the available types of computer displays. Smaller displays (such as a single laptop monitor) limit how much information is visible at a time and cannot display full-size items in a spatial presentation. Though costly, larger displays allow for persistent spatial layouts of information to help users visualize relationships among pieces of information. It is unclear, however, what properties of a spatial presentation are most important. Do the benefits of a spatial layout depend on display size? Is location alone sufficient to provide benefits for cognitive processing, or is persistent visibility also required for an effective spatial information presentation?

Without persistent visibility, small displays often rely on dynamic visualizations to deliver large amounts of information within a limited amount of space. Visualizations can change over time, but different learners require different amounts of time to process the same information. Interactivity can help by allowing learners to control the delivery of information, easing the demand on working memory [8]. Interactive viewing allows learners to control the order and duration of information viewing, but with the added costs of additional decision-making and view-manipulation tasks.

Our investigation considers how interactivity and spatial distributions of information affect learning outcomes and learner strategies when learning with visual presentations. We present the results of a controlled study that evaluated comprehension and detail recall on a pictorial learning activity. We predict that the benefits of large displays stem from both persistent visibility and the distinct locations where information is presented. In our study, we controlled both dimensions in order to isolate their individual effects and gain a deeper understanding of how users use space to aid learning and information processing.

In the first part of the study, we prevent persistent visibility and focus on the effects of location distribution. We compared performance between a distributed presentation among multiple monitors (see Figure 1) and a slideshow-style presentation on a single monitor (see Figure 2). Additionally, we studied the relationship 
between this presentation style and the method of view controlautomatic or interactive. The results show that learners performed significantly better with the simpler slideshow presentation than with a spatially-distributed layout, suggesting that-without visible persistence-spatial information presentations can have negative effects on cognitive processing.

In the second part of the study, we evaluated learning performance based on a standard distributed layout with persistent visibility. Learning scores using this presentation were significantly higher than the distributed presentations lacking persistence. These results demonstrate the importance of persistent visibility and suggest that greater spatial variability alone is not enough to support advantages for learning. Further, if the benefits of spatial distributions with large displays stem from the ability to easily access visibly persistent information, this implies the benefits of a spatial distribution may diminish on smaller displays that lack persistence and rely on virtual panning. This research provides new insights into how space and locations are used during learning activities on large display workstations. While previous studies have shown evidence of cognitive benefits due to spatial variance (e.g., [22] [9]), our results suggest a limit to which increasing the spatial variance of information locations supports improvements.

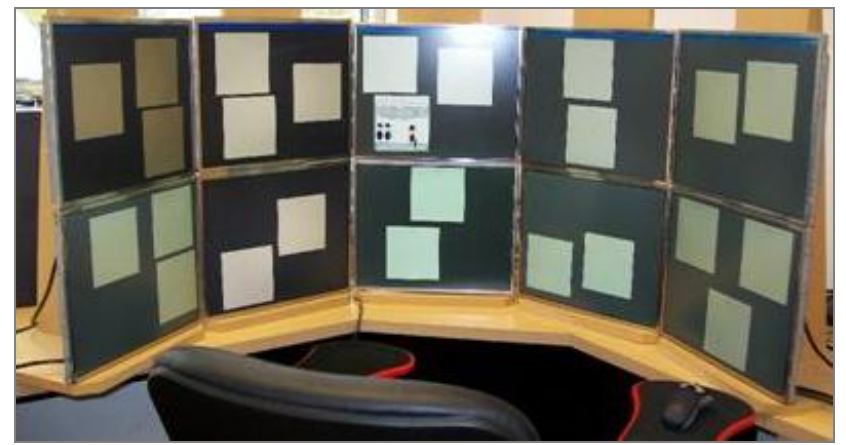

Figure 1. The ten-display workstation with the distributed layout condition.

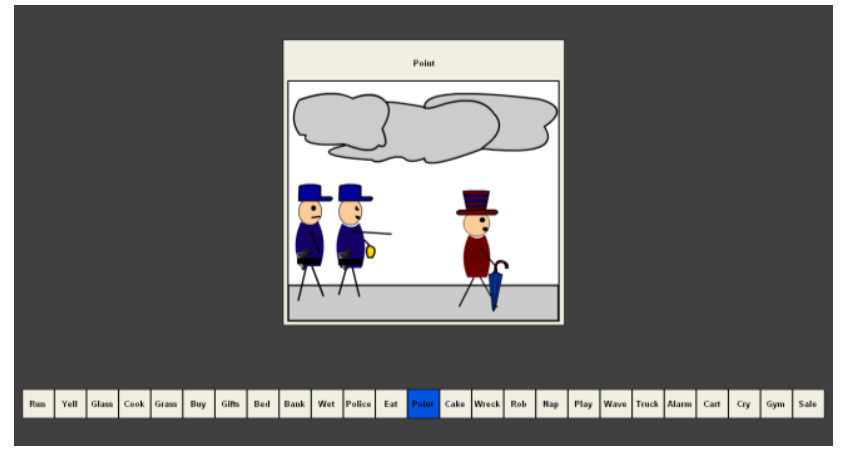

Figure 2. The slideshow layout. All images are viewed at the same location on a single monitor. The title of the current card is highlighted in the list below the card.

\section{RELATED WORK}

Visualization has a long history of success in helping users to understand information [6]. Whether used to represent complex information in a unique way, communicate the meaning of information to others, or serve as a work space for problem solving, many visualizations are designed for the purpose of helping viewers learn new information. Discussing the importance of comput- er-supported data visualization, Card, Mackinlay, and Shneiderman [6] describe the primary role of interactive visualization as the amplification of cognition. With a similar perspective, Norman refers to visualizations as cognitive artifacts, serving as a means of offloading cognitive processing into the world [18, 29]. Graphical multimedia allows learners to externalize their internal representations of information, reducing the strain on working memory [24, 29]. As a result, more cognitive effort can be used for constructing new internal knowledge structures, sense-making, or other information processing tasks. By this model, new information will be more efficiently understood and encoded in long term memory [17]. It is not surprising, then, that presentation design is important for learning and sense-making. Educational multimedia is often used to present information through the combination of multiple representations, providing the potential to aid learning by allowing learners to experience related information in an integrated context [13]. Our research investigates the roles of spatial layouts and interactivity when learning through visualization.

\subsection{Supporting Learning with Space}

Spatial grouping is a common way to effectively present information and support understanding [12]. Studying educational multimedia, Mayer [16] presented evidence that students learned more effectively and demonstrated more creative problem-solving when related text and images were presented in the same location, rather than separated into different regions.

Similar to the theory that information is processed using multiple types of mental resources, it has been proposed that knowledge items are stored not as single units, but as collections of features (e.g., [5, 27]). Brown and McNeill [5] suggest that memories of items are organized by their features, and sometimes certain features are able to be recalled individually. Research has also shown evidence that recollection of features can serve as a cue to aid the retrieval of associated information [e.g., 26].

Human cognition processes different information features in different ways. While some features of information are learned through effortful processing, requiring attention and intentional learning, other types, such as spatial or temporal information, can be processed automatically, requiring little or no extra attention or demand on available cognitive resources [7]. While a variety of types of information (e.g., images, words, sounds) can be used to cue retrieval, our research focuses on spatial cues. Mandler, Seegmiller, and Day [14] provided strong evidence that spatial information is learned automatically, even when the learner is focused on other features. Consequently, location could be an ideal candidate as a form of redundant coding to reinforce learning and to aid the recall of other information features. By Pylyshyn's model of spatial indexing, locations can be used as references to other information that is not visible [21]. That is, by referencing a location as an index, it is possible to recall the information that was associated with that index.

Numerous studies have provided evidence that people do refer to spatial information when recalling information. In one such study, Richardson and Spivey [23] asked participants to recall details about objects that were no longer visible. The study found that participants often looked to the locations where the objects were when answering questions about those objects - even though the relevant information was no longer there. In research on menu design, Kaptelinin [11] concluded that software users rely on locations in lists more than on textual descriptors when selecting menu 
items. Rather than simply reading the text of the list items, participants selected items based on their positions, relying on a mapping of items to locations. Providing further evidence of the benefits of using spatial information for information processing, research by Hess, Detweiler, and Ellis [9] found memory benefits when location was used as a redundant indicator. The researchers found performance improvements on a memory task when information was correlated with positions in a grid layout, rather than displayed in the same location. We found a similar effect in a previous study [22] using a sequence memorization task. This study found that participants remembered significantly more items when information was displayed at different locations, rather than in the same place. These results demonstrate the value of spatial indexing for data retrieval.

\subsection{Interactive and Active Learning}

The rationale for using interactive multimedia to assist learning is largely based on the theory that knowledge is gained through active experience. Piaget proposed that learning was a process of discovery, and that individuals construct and organize mental knowledge structures based on their experiences [19]. In addition to providing a means for to actively explore information, interactive graphical multimedia allows learners to represent and experience their internal knowledge structures visually [24, 29]. Interactive exploration allows learners to experience information in multiple ways, supporting the construction and testing of various knowledge representations [20]. The goal is to support more meaningful learning through connections with related pieces of information [17], as meaningful material can more effectively be learned and recalled [e.g., 10, 15]. Researchers have shown that interactive visualizations can improve problem solving and creativity [25], as well as understanding [3].

With our study, we expand upon the body of knowledge about interactive learning and the use of space during cognitive processing. In the first part of the experiment, we focus on spatial presentations without persistent visibility in order to isolate the effects of locations alone. We also studied the effects of interactive viewing on learning outcomes and learner strategies. With the second part of the experiment, we studied how persistent visibility affects learning.

\section{EXPERIMENT: PART I}

We conducted a controlled experiment to investigate how learning performance and learner strategies are affected by: (1) the spatial distribution of information in a visual presentation, and (2) interactive control over information viewing.

\subsection{Hypotheses}

Past research has found evidence that users externalize memory and thought into space while using interactive, large-display systems to analyze information [e.g., 2]. We hypothesized that a distributed spatial layout would support superior learning performance due to the increased variety in available positional cues.

Further, we hypothesized that interactive, user-controlled viewing would improve task performance. We expected that interactive view control would improve learners' abilities to map information to locations in space, enabling the use of spatial indexing as a memory aid. We suspected that the added element of interactivity would allow users to give further meaning to the space, strengthening the effectiveness of the information mapping.

\subsection{Task}

To test our hypotheses, we designed a story task to evaluate both comprehension and detail recall. Participants viewed a set of 25 event cards. The cards included simple, graphical representations of nine visually distinct characters in various situations along with single-word titles to describe the event. Figure 3 shows samples of card images. The cards portrayed simple events with the same characters so that sequences of events could be interpreted as short sub-stories. Additionally, individual characters and events contributed to multiple sub-stories, causing significant overlap among sub-stories and allowing the entire collection of events to be interpreted as a single large, complex story. The primary story subplots include: a car accident, a store robbery, shopping, a birthday party, and a broken window. Participants were asked to determine the story and sub-stories based on the events viewed in the cards.

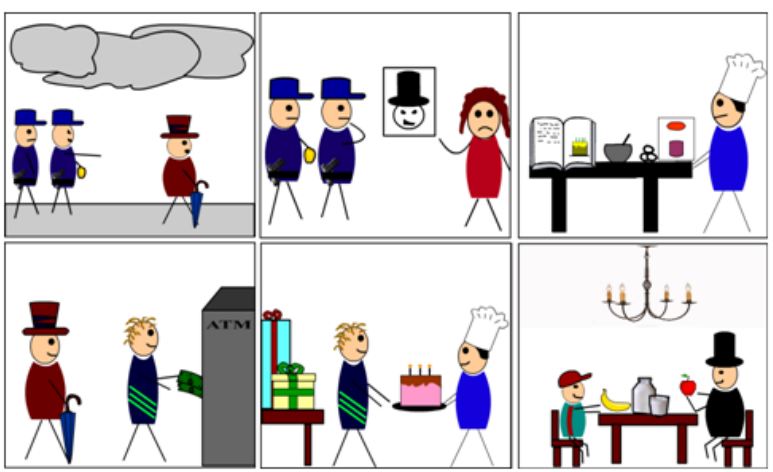

Figure 3. Examples of images used in the dataset. Starting from the upper left corner and moving clockwise, the titles of these cards are: Point, Police, Cook, Eat, Cake, and Bank.

The size of the data set and the complexity of the overlapping substories were determined through a series of small pilot studies. Because this was a controlled experiment and the total viewing time was held constant (as explained further in the Design section), images were chosen over purely textual information in order to avoid confounds due to participants' different reading speeds. The data set was designed to support questions of both memory of details and understanding of story events. The question set and scoring criteria focused more on the more significant events for the main story plots (e.g., the robbery or car crash) than on less significant events (e.g., mowing the lawn or walking the dog).

After viewing the event cards, participants were asked a series of questions to test their knowledge and understanding of the presented information. Participants' verbal responses were videotaped in order to aid scoring based on a prepared rubric. Questions were designed to evaluate comprehension of the meaning of the events and stories as well as simple detail recall. For questions focusing on memory of details, participants earned points for correctly recalling characters and details from the events shown in the story cards. Examples of a detail recall questions include:

- What food products were present in the "Eat" scene?

- What character or characters were in the "Gym" scene?

Other questions evaluated comprehension, involving understanding of the meaning of the events and stories. These questions required participants to do more than simply recall the images on the panels. To earn points for these questions, participants were required to explain connections among the characters, explain what caused events to occur, or hypothesize future events and appropri- 
ate emotional states of the characters. Examples of comprehension questions are:

- Can you come up with a sub-story of events that link the boy with a red baseball cap to the man with an umbrella?

- How would you expect the man with a black hat to be feeling at the end of the day, and why?

- Describe an event or scene that you would expect the man in the black hat to be doing after the events shown in the story.

Scoring was calculated in accordance with a pre-constructed rubric, with separate scores calculated for detail recall and comprehension questions. The total score was based on all questions.

\subsection{Design}

Participants viewed the event cards on a ten-monitor display, configured in a curved $120^{\circ}$ arc with a $2 \times 5$ arrangement (see Figure 1). Each monitor was 17 inches with $1280 \times 1024$ resolution. Participants sat in a swivel chair in front of the display. Because each participant could only complete the learning task a single time, viewing mode and presentation style were varied in a $2 \times 2$ between-subjects design. The two viewing modes we tested were automatic and interactive control, and the two presentation styles were slideshow and distributed layout.

Only one card image was ever visible at a time. In the slideshow layout, all cards were presented in the same location-on a single monitor directly in front of the participant (see Figure 2). Below the location where the cards were shown, a horizontal list always showed all textual titles. In the distributed presentation style, the cards were distributed across all monitors of the display so that every card had its own persistent location (see Figure 1). While only a single card image was shown at a time, the locations of all cards were always visible as empty boxes with the textual titles visible. In this way, both presentation conditions always had all titles visible and provided a spatial location corresponding to each image; however, these locations had much higher spatial variance in the distributed layout where the images themselves were displayed in different locations. This allowed us to isolate the effects of spatial location without the confounding effects of persistent visibility that is afforded by normal large-display workspaces. For both presentation styles, the cards were ordered or arranged in the same predetermined organization - events were jumbled so that the stories were not presented in chronological order.

In the automatic presentation conditions, each card image was displayed for five seconds before it was hidden and the next image was displayed. Every card was shown twice in this fashion. In the slideshow condition, all cards showed up at the same location, but card order progressed from left to right through the list of titles. Cards were also displayed from left to right (in the same order) for the distributed conditions. Participants had no way of interacting or controlling the view of the cards.

In the interactive control conditions, each participant used a mouse to manually control the order and duration in which card images were viewed. The participant could make a card image visible by moving the mouse cursor over its title in the slideshow list or over its card in the distributed layout. The total amount of viewing time was limited to 250 seconds - the same as in the automatic conditions.

In all conditions, images and titles were hidden when the viewing period ended, but blank cards or labels remained on the display as placeholders for where the information was displayed. That is, for the distributed layout, blank cards were left on the display for the remainder of the evaluation; for the slide-show layout, empty blocks were still visible in the title list (but with no text).

We studied the effects of viewing mode and presentation style on a variety of metrics. In addition to learning scores, we also asked each participant to report a percentage of confidence of story comprehension. We also measured location recall by having participants point to the blank placeholders and report what event was shown at each location. Video recording and eye tracking were used to aid scoring and to study participant strategies.

\subsection{Procedure}

After having participants complete a general demographic questionnaire, we explained the task and the display system with the aid of a prepared script. Using a small set of event cards with no information relating to the actual story of the primary task, we provided a brief demonstration of how they would be viewing the story images. For participants in the interactive viewing conditions, this included practice using the mouse. We again reviewed the purpose of the task, asking participants to identify the stories and encouraging them to pay attention to how the characters are connected. Participants were informed that the images were jumbled and the image organization was independent of the chronology of the story.

Participants then viewed the cards in the manner determined by their experimental condition. Immediately after the viewing phase, we verbally administered a portion of a number-memorization test to help clear working memory of information about the story set before our real questions. For this test, we verbally listed a sequence of numbers and then asked participants to write down those numbers. This task took approximately one minute, helping to establish that the following questions would be answered based on information from long-term memory (memory research has found that retention in working memory is generally limited to around ten to fifteen seconds without active rehearsal [e.g., 4]).

We then verbally asked the questions for the evaluation of learning. Next, we asked participants to describe their strategies and thought processes when viewing the information and when answering the questions. Finally, referring to the blank placeholders remaining on the display, we asked participants if they remembered any of the corresponding events for the locations.

\subsection{Participants}

Thirty-two undergraduate students participated in Part I of the study. An equal number of male and female students participated, with gender balanced across conditions. Participants came from a variety of academic disciplines, also balanced as well as possible. Ages ranged from 18 to 23 years with a median age of 20 years.

\subsection{Results}

Because Shapiro-Wilk tests of normality for each of our metrics suggested that they were normally distributed, we were able to use two-way factorial analyses of variance (ANOVA) to analyze the results. We performed multiple analyses to test the effects of presentation style and viewing method on various performance outcomes. The analysis for total learning score showed a significant main effect of presentation layout on overall scores, with $\mathrm{F}(1$, $28)=10.21$ and $p<0.005$. Total scores were significantly better with the slideshow-style presentation $(\mathrm{M}=74.19, \mathrm{SD}=19.44)$ 
than with the distributed layout $(\mathrm{M}=51.31, \mathrm{SD}=20.00)$. This is the opposite of the hypothesized effect of presentation layout.

The same effect was also found for the ANOVA test for comprehension scores. Comprehension scores were significantly better with the slideshow-style presentation $(\mathrm{M}=50.00, \mathrm{SD}=16.31)$ than with the distributed layout $(\mathrm{M}=32.63, \mathrm{SD}=16.19)$, with $\mathrm{F}(1,28)=9.76$ and $\mathrm{p}<0.005$

For detail recall, scores were better with the slideshow-style presentation $(\mathrm{M}=24.19, \mathrm{SD}=4.32)$ than with the distributed layout $(\mathrm{M}=20.56, \mathrm{SD}=6.17)$, but this was not significant at $\mathrm{p}<$ 0.05 level. However, with $\mathrm{F}(1,28)=3.53$ and $\mathrm{p}=0.07$, we suspect that this effect would have been significant with more trials.

No significant effects on learning scores were found due to viewing mode, with $\mathrm{F}(1,28)=0.04$ for total scores, $\mathrm{F}(1,28)=0.02$ for comprehension scores, and $\mathrm{F}(1,28)=0.42$ for detail recall scores. No significant interactions were found between viewing mode and presentation layout for learning scores, with $F(1,28)=0.42$ for total scores, $\mathrm{F}(1,28)=0.10$ for comprehension scores, and $\mathrm{F}(1$, 28) $=0.21$ for detail recall scores.

An analysis also found a significant effect on location recall (the number of event locations that participants could correctly recall after the questions) due to presentation style, with $\mathrm{F}(1,28)=$ 14.70 and $p<0.001$. This showed that participants were better able to remember the associated locations for events with the distributed layout $(\mathrm{M}=9.56, \mathrm{SD}=3.54)$ than in the slideshow presentation $(M=5.19, S D=2.93)$. Location recall was not significantly affected by viewing mode, with $\mathrm{F}(1,28)=0.01$, and no interaction was found between presentation style and viewing mode, with $\mathrm{F}(1,28)=2.35$.

The analysis of confidence of comprehension did not show significant differences due to presentation style, with $\mathrm{F}(1,28)=3.00$, or viewing mode, with $F(1,28)=0.03$. However, the test did show a trend with confidence levels being higher for the slideshow presentation $(\mathrm{M}=63.81, \mathrm{SD}=19.32)$ than the distributed layout $(\mathrm{M}=49.44, \mathrm{SD}=25.99)$, with $\mathrm{p}=0.09$.

\subsection{Discussion}

While we had hypothesized that participants would achieve higher learning scores with the distributed layout, this was clearly not the observed outcome. Learning scores were significantly lower in the distributed layout than in the slideshow-style presentations.

The location recall results indicate that participants were better able to remember the associated locations for event cards with the distributed layout. However, the performance results suggest that these additional location memories did not support performance improvements, despite the fact that many participants were referring to locations to aid recall during questioning (a more detailed presentation of participant strategies is given in Part II).

These were surprising results, as previous research with spatial separation found the opposite effect $[9,22]$. As no interactions were observed between presentation style and viewing mode, the results suggest that users did not suffer from problems interacting with the mouse in a larger space. We also know that the results were not due to poor spatial memory since participants had better memory of locations in the distributed layout conditions.

One possible explanation is that participants performed better with the slideshow presentation due to higher familiarity with similar presentation styles (e.g., viewing PowerPoint slides, web browsing with multiple tabs, switching among multiple open documents or applications on a single monitor). Alternatively, it could be that it takes practice to establish effective spatial strategies when using larger workspaces; we leave this to future work.

Another explanation - and our current hypothesis - is that perhaps spatial mappings are only useful when the locations carry meaning for the data. That is, the results could be different if the information was spatially grouped with some meaningful organization, such as by chronology or by characters. Because card placements were jumbled in our organizations, locations did not provide additional organizational cues. In future work, we plan to further investigate the relationship between the use of locations and meaningful spatial organization.

As there were no significant differences due to viewing mode, we reject the hypothesis that interactive viewing enables learning improvements. This result has important educational implications, providing evidence that simply adding interactivity does not guarantee learning benefits. Further, because the location recall results showed no effects due to the presence or absence of interactivity, we reject the claim that interactive viewing gives additional meaning to locations or makes information locations easier to remember. It could be that view control is not a complex enough type of interactivity to add meaning to a location. Another possibility is that viewing mode had little effect due to the relatively small size of the data set or the relatively short viewing time.

After Part I of the experiment, it was unclear how learning performances would compare with a standard spatial distribution. As all conditions in the first part of the experiment allowed participants to view only one image at a time, the distributed layout presentations lacked the persistent visibility of information that is normally available with spatial layouts on large-displays. By intentionally crippling persistence in the distributed layouts, we were able to isolate effects due to spatial locations. But how much does persistent visibility really affect the use and benefit of a spatial layout? To address this question, we expanded the experiment by adding an extra condition to help investigate whether learners would take advantage of the distributed layout if all information were visible at all times.

\section{EXPERIMENT: PART II}

The first part of the experiment focused on studying learning differences due to varying levels of spatial distribution without persistent visibility. In the second part, we extended the experiment to study how persistent visibility affects learning performance and learning strategies. By maintaining the same design and evaluation as used in Part I, we were able to add an additional condition to further our investigation of how learners use spatial presentations to learn and understand new information.

\subsection{Hypotheses}

We hypothesized that a distributed presentation with persistent information visibility would allow learners to use the locations of the spatial layout to help organize information and aid recall. Thus, we expected the addition of persistent visibility to lead to better learning scores than achieved in the distributed presentations from Part I. Also, due to the ability to view and compare multiple images at the same time, we expected performance improvements over the slideshow-style presentations. 


\subsection{Design}

For part II of the study, we ran one new condition to compare to the results from Part I. Thus, Part II used the same experimental task, procedure, and evaluation metrics as Part I. Ten undergraduate students (three males and seven females, ages 18 to 21 ) from various academic disciplines participated in the new condition. Thus, combined with the 32 participants from Part I, the full experiment had a total of 42 participants.

The new condition used a distributed layout with the same organization as the distributed layouts of Part I. However, instead of having only one image visible at a time, as with the automatic and interactive presentations, all card images were always visible for the duration of the viewing phase. As with the conditions in Part I, a 250 second time limit was enforced. Also as in the previous conditions, the images and titles were hidden when the time limit was reached, leaving only blank cards on the display.

\subsection{Results}

We analyzed the results by considering learning scores, memory of locations, and participant strategies for all conditions from Part I and Part II of the experiment.

\subsubsection{Learning Performance}

To analyze performance results, we treated each of the four conditions from Part I as a separate group and added the new condition from Part II, giving us five distinct presentation conditions. We again tested each of our metrics for normality with Shapiro-Wilk tests, finding that the learning scores were approximately normally distributed. We tested for differences in learning scores among the five conditions with a one-way independent ANOVA for each score category (total score, comprehension, and detail recall).

The analysis for total scores found a significant main effect due to viewing condition, with $\mathrm{F}(4,37)=3.54$ and $\mathrm{p}<0.05$. Figure 4 shows means and standard deviations for total scores. A post-hoc Student's t-test revealed that scores for the persistent-visibility distributed condition and both the slideshow conditions were significantly higher than the automatic and interactive distributed conditions. The post-hoc test did not show a significant difference between the automatic and interactive distributed conditions.

The analysis for comprehension scores also revealed a significant main effect with $F(4,37)=3.13$ and $p<0.05$. As with the total scores, a post-hoc Student's t-test showed that scores for the persistent-visibility distributed $(\mathrm{M}=49.10, \mathrm{~S}=8.94)$, the automatic slideshow $(M=50.50, S=17.11)$, and the interactive slideshow $(\mathrm{M}=49.50, \mathrm{~S}=16.63)$ conditions were significantly higher than the automatic $(\mathrm{M}=31.25, \mathrm{~S}=17.40)$ and interactive $(\mathrm{M}=34.00$, $\mathrm{S}=15.95)$ distributed presentation conditions.

Finally, with $\mathrm{F}(4,37)=1.10$ and $\mathrm{p}=0.37$, the analysis for detail recall scores did not show a significant effect.

\subsubsection{Location Recall}

Figure 5 shows average location recall scores for all conditions. We tested for differences in location recall among the five conditions with a one-way independent ANOVA. The analysis showed a significant main effect with $\mathrm{F}(4,37)=7.42$ and $\mathrm{p}<0.0005$. A post-hoc Student's t-test showed that location recall for all distributed layouts was significantly higher than the slideshow conditions. Though the persistent-visibility distributed layout did have the highest overall location recall scores, scores were not significantly different among the three distributed layout conditions.

\subsubsection{Learning Strategies}

We also studied participant learning strategies by analyzing standard video recordings, eye-tracking video, and interview responses. We considered two general types of strategy classification: viewing order and intentional use of locations.

For this activity, viewing order is the order in which card images were viewed during the learning session. Participants in the automatic presentation conditions were not able to control the viewing order because the images were shown to them automatically. In both interactive conditions and in the persistent-visibility distributed condition, participants were able to choose the viewing order. Most participants (73\%) from these conditions employed the same general type of viewing strategy. At the beginning of the viewing session, these participants first briefly scanned over the entire data set in an attempt to get an overview of all cards. They then began to search for and focus on specific images based on logical story constructs (e.g., time of day, same characters). Other participants (23\%) did not spend any time scanning the entire dataset, and immediately began trying to search for and match events and characters. One participant from these conditions (specifically, in the interactive distributed layout) never used a search-and-match type viewing strategy, but instead continually scanned over the entire card set for the duration of the viewing time.

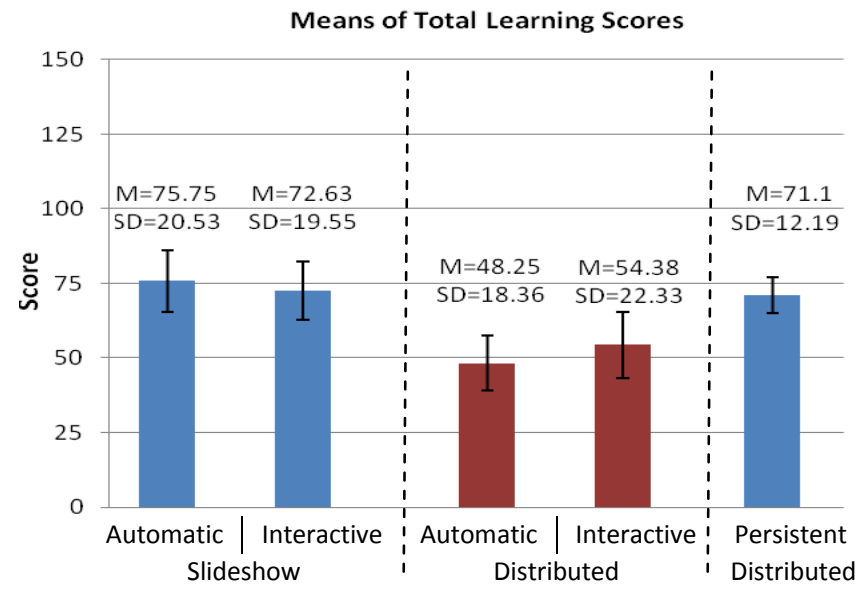

Figure 4. Means of total learning scores with standard deviations after Part II. Different colored bars are significantly different. Scores for the persistent-visibility distributed and both slideshow conditions are significantly better than both the automatic and interactive distributed conditions.

We also considered intentional use of locations during the task. That is, we studied whether or not participants intentionally attempted to use locations to aid in their learning or recall. This was determined through the post-study interview, in which we asked participants if they tried to use the locations during the learning or questioning periods. Note that the slideshow presentation still supported the use of locations due to the inclusion of the title list below the image presentation area (see Figure 2).

Figure 6 shows the percentages of participants that intentionally used locations for each condition. While the data do not meet the assumptions of a chi-square test for a formal analysis, the percentages do suggest that conditions did affect spatial strategies. The highest percentage of participants intentionally used locations in 
the persistent-visibility distributed condition, while the interactive slideshow condition had the lowest overall percentage.

We believe that the difference between automatic and interactive slideshow conditions can be explained by differences in viewing order. In the automatic slideshow presentation, the images were always presented in a linear progression. In a way, this presentation method forced participants to relate the images to their associated locations in the list. With the interactive slideshow, on the other hand, participants were able to continuously slide the mouse cursor over the list - without paying attention to location - until they found the desired image.

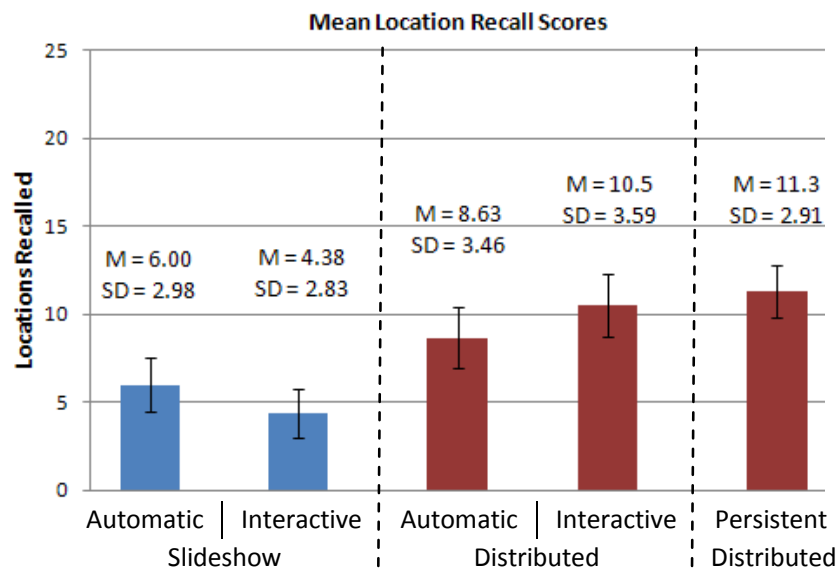

Figure 5. Mean location recall scores. Different colored bars are significantly different. Location recall was higher with all distributed layouts than with the slideshow presentations.

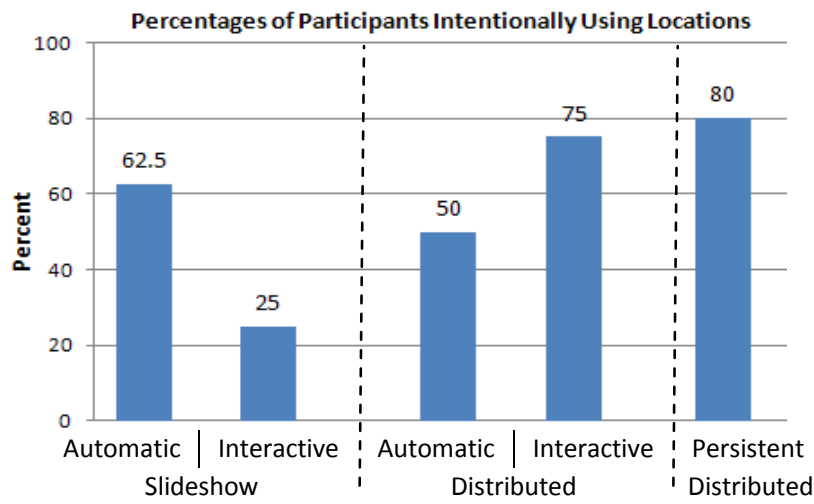

Figure 6. Percentages of participants in each condition that intentionally used locations to aid learning or recall.

\subsection{Discussion}

As hypothesized, the results from Part II confirm that learning scores with the persistent-visibility distributed layout were superior to the automatic and interactive distributed presentations. These results demonstrate the value of persistent visibility in largedisplay systems. Our strategy analysis helps further explain this benefit. Most participants (all but one) in the interactive or persistent-visibility conditions spent considerable time searching for specific events or characters in the data set. This certainly is faster and easier when all images are visible at the same time.

Learning scores in the persistent-visibility distributed condition were not significantly different from the slideshow conditions. Thus, for this task, we reject the hypothesis that a distributed presentation with persistent visibility supports greater learning than the slideshow style presentation. These results indicate the importance of presentation design. The experiment clearly demonstrates that a distributed spatial layout is not always an automatic method of improving cognitive processing.

Of course, this does not necessarily mean that a spatial largescreen presentation cannot support improved processing and learning of information. It is possible that our evaluation was not sensitive enough to detect differences. If participants were approaching the limit of how much could be learned in our task, this could explain why scores in the persistent-visibility condition were not significantly different. Similar experiments with larger data sets and longer learning sessions would be helpful in further investigating differences due to varying spatial presentations.

Additionally, as we discussed in the Discussion section for Part I, it is still unknown whether these results would differ for logically organized information layouts rather than random, jumbled organizations. If the information was meaningfully organized in space, then learners' better recall for location could potentially be used to aid information recall. This is a matter for future investigation.

The location recall results do serve as further evidence that locations can be learned automatically. However, memorization of locations was not always purely incidental with our task, as many participants did consciously pay attention to locations during learning. Still, others who intentionally attempted to use locations during questioning reported that they had not paid attention to locations during the viewing session. Also note that the results of Part II provide evidence that greater memory of locations does not negatively interfere with learning. Though the results of Part I showed that participants in the distributed conditions had higher location recall scores and lower learning scores, Part II showed that participants were able to achieve higher learning scores while still having high location recall scores.

The results from the persistent-visibility distributed condition show that learners achieved relatively high learning scores while also demonstrating high location recall, suggesting that the memory of spatial information did not interfere with the memory or understanding of the story information. Thus, designers should still consider presenting information spatially if the spatial organization can support the logical organization of the content.

\section{CONCLUSION AND FUTURE WORK}

While previous studies have found that additional spatial cues have the potential to aid memorization and learning (e.g., $[9,22]$ ), our research suggests that-contrary to what was expectedincreasing the spatial variance of information locations does not necessarily support cognitive processing. Though displaying information in spatially distributed layouts helped participants to better recall information locations, learning performance was negatively affected. This suggests that participants were unable to take advantage of their knowledge of locations to aid their learning. This disadvantage was eliminated when learners were permitted constant visibility of information, indicating the high importance of persistent visibility when working with large-displays. This suggests that spatial layouts on small displays (lacking persistent visibility and relying on virtual navigation) would lose the benefits of the spatial layout.

These results have provided the foundation for an extended research plan for studying the effects of spatial information distributions with large display systems. Future work includes considera- 
tions for the size of the data set, type of data representation, organization of information, and type of interactivity. We believe that the size of the data set may affect the use of spatial cues when learning. With a larger dataset, interactive view control may become necessary as users need to refer back to information on demand. Additionally, as our experiment provided jumbled presentations in order to isolate the effects of spatial mapping, the results have informed plans for future studies of varying organizational schemata. We hypothesize that logically organized information layouts could give meaning to the locations in space and allow learners to take advantage of their memories of locations for learning. Another question is whether similar results would be observed with different data representations. Our task was based primarily on graphical information that participants viewed and integrated into stories. It is possible that different results could be observed with data sets with different formats, such as textual information, rather than primarily graphical representations.

We plan to extend this research to study additional forms of interactivity, such as organization or annotation. It is possible that view control may not be a complex enough type of interactivity to help learners to connect information to its location. We are interested in comparing different interaction types.

\section{REFERENCES}

[1] Anderson, J. R. and Schunn, C. D. Implications of the ACTR Learning Theory: No Magic Bullets. Advances in Instructional Psychology, Volume 5: Educational Design and Cognitive Science (2000), 1.

[2] Andrews, C., Endert, A. and North, C. Space to think: large high-resolution displays for sensemaking. Proceedings of ACM CHI (2010).

[3] Bodemer, D., Ploetzner, R., Feuerlein, I. and Spada, H. The active integration of information during learning with dynamic and interactive visualisations. Learning and Instruction, 14, 3 (2004), 325-341.

[4] Brown, J. Some Tests of the Decay Theory of Immediate Memory. Quarterly Journal of Experimental Psychology, 10 (1959), 12-21.

[5] Brown, R. and McNeill, D. The" tip of the tongue" phenomenon. Journal of verbal learning and verbal behavior, 5, 4 (1966), 325-337.

[6] Card, S. K., Mackinlay, J. D. and Shneiderman, B. Readings in information visualization: using vision to think. Morgan Kaufmann, (1999).

[7] Hasher, L. and Zacks, R. T. Automatic and effortful processes in memory. Journal of Experimental Psychology: General, 108, 3 (1979), 356-388.

[8] Hegarty, M. Dynamic visualizations and learning: getting to the difficult questions. Learning and Instruction, 14, 3 (2004), 343-351.

[9] Hess, S. M., Detweiler, M. C. and Ellis, R. D. The Utility of Display Space in Keeping Track of Rapidly Changing Information. Human Factors: The Journal of the Human Factors and Ergonomics Society, 41, 2 (1999), 257-281.

[10] Johnson, R. E. Meaning in Complex Learning. Review of Educational Research, 45, 3 (1975), 425-459.
[11] Kaptelinin, V. Item recognition in menu selection: the effect of practice. ACM INTERACT '93 and CHI '93 conference companion on Human factors in computing systems, (1993).

[12] Larkin, J. H. and Simon, H. A. Why a diagram is (sometimes) worth ten thousand words. Cognitive science, 11, 1 (1987), $65-100$

[13] Levie, W. and Lentz, R. Effects of text illustrations: A review of research. Educational Technology Research and Development, 30, 4 (1982), 195-232.

[14] Mandler, J. M., Seegmiller, D. and Day, J. On the coding of spatial information. Memory \& Cognition, 5,1 (1977), 10 16.

[15] Mayer, R. E. Integration of information during problem solving due to a meaningful context of learning. Memory \& Cognition, 4, 5 (1976), 603-608.

[16] Mayer, R. E. The promise of multimedia learning: using the same instructional design methods across different media. Learning and instruction, 13, 2 (2003), 125-139.

[17] Mayer, R. E. and Moreno, R. Nine ways to reduce cognitive load in multimedia learning. Educational psychologist, 38, 1 (2003), 43-52.

[18] Norman, D. A. Cognitive artifacts. Designing interaction: Psychology at the human-computer interface (1991), 17-38.

[19] Piaget, J. The development of thought: the equilibrium of cognitive structures. Viking Press., New York (1977).

[20] Price, S. and Rogers, Y. Let's get physical: the learning benefits of interacting in digitally augmented physical spaces. Computers \& Education, 43, 1-2 (2004), 137-151.

[21] Pylyshyn, Z. The role of location indexes in spatial perception: A sketch of the FINST spatial-index model. Cognition, 32, 1 (1989), 65-97.

[22] Ragan, E. D., Bowman, D. A. and Huber, K. J. Supporting Cognitive Processing with Spatial Information Presentations in Virtual Environments. Virtual Reality (2012; to appear).

[23] Richardson, D. C. and Spivey, M. J. Representation, space and Hollywood Squares: looking at things that aren't there anymore. Cognition, 76, 3 (2000), 269-295.

[24] Scaife, M. and Rogers, Y. External cognition: how do graphical representations work? International Journal of HumanComputer Studies, 45, 2 (1996), 185-213.

[25] Tennyson, R. D. and Breuer, K. Improving problem solving and creativity through use of complex-dynamic simulations. Computers in Human Behavior, 18, 6 (2002), 650-668.

[26] Tulving, E. and Osler, S. Effectiveness of retrieval cues in memory for words. Journal of experimental psychology, 77, 4 (1968), 593-601.

[27] Wickens, D. D. Encoding categories of words: An empirical approach to meaning. Psychol. Rev., 77, 1 (1970), 1-15.

[28] Wise, J. A., Thomas, J. J., Pennock, K., Lantrip, D., Pottier, M., Schur, A. and Crow, V. Visualizing the non-visual: spatial analysis and interaction with information from text documents. Proceedings of IEEE Symposium on Information Visualization. (1995).

[29] Zhang, J. and Norman, D. A. Representations in distributed cognitive tasks. Cognitive science, 18, 1 (1994), 87-122. 